\title{
FACTORING ROSENTHAL OPERATORS
}

\author{
TERESA ALVAREZ
}

\begin{abstract}
In this paper we show that a Rosenthal operator factors through a Banach space containing no isomorphs of $l_{1}$.
\end{abstract}

All spaces are to be Banach spaces. If $X$ is a Banach space, $X^{n}$ denotes the bidual of $X, J_{X}$ is the canonical isometry embedding $X$ in $X^{\prime \prime}, B_{X}$ is the closed unit ball of $X$ and $I_{X}$ denotes the identity operator on $X$. Let $\mathrm{L}(X, Y)$ be the set of all continuous linear operators from $X$ into $Y$.

Recall that an operator $T \in \mathrm{L}(X, Y)$ is said to be completely continuous if every weakly convergent sequence $\left(x_{n}\right)$ is mapped into a norm convergent sequence $\left(T x_{n}\right)$. The class of all completely continuous operators from $X$ into $Y$ is denoted by $\mathrm{Cc}(X, Y)$ and $\mathrm{Co}(X, Y)$ will denote the space of all compact operators from $X$ into $Y$.

Let $T \in \mathrm{L}(X, Y)$. Then $T$ is called a Rosenthal operator if $S T \in \operatorname{Co}\left(X, Y_{0}\right)$ for all $S \in \operatorname{Cc}\left(Y, Y_{0}\right)$, where $Y_{0}$ is an arbitrary Banach space. Using a theorem which is due to Rosenthal [8] and Dor $[2]$ in the real and complex case, respectively, one gets that $T$ is a Rosenthal operator if and only if it maps bounded sequences into sequences possessing weak Cauchy subsequences (see for example [6]), and hence $T$ is a Rosenthal operator if and only if $T\left(B_{X}\right)$ is $\sigma\left(Y^{\prime \prime}, Y^{\prime}\right)$-relatively sequentially compact in $Y^{\prime \prime}$.

The Rosenthal operators are called weak Cauchy operators by [5] and conditionally weakly convergent operators by $[\mathbf{7}]$.

An operator $T \in \mathrm{L}(X, Y)$ is called a $\ell_{1}$-singular operator if for each $S \in$ $\mathrm{L}\left(\ell_{1}, X\right)$, the composition $T S$ is not an isomorphic embedding [5]. So, $T \in$ $\mathrm{L}(X, Y)$ is a Rosenthal operator if and only if is a $\ell_{1}$-singular operator. $\operatorname{Ro}(X, Y)$ will denote the class of all Rosenthal operators from $X$ into $Y$.

Let $N \ell_{\perp}$ be the space ideal of all Banach spaces containing no isomorphic copy of $\ell_{1}$ and let $\operatorname{Op}\left(N \ell_{1}\right)$ be the operator ideal of all operators which factor through spaces in $N \ell_{1}$.

In this note we shall prove that $O p\left(N \ell_{1}\right)$ coincides with the class of all Rosenthal operators. For this end we shall use the construction of DaviesFigiel-Johnson-Pelczynski [1]. Let $W$ be a convex, symmetric and bounded 
subset of a Banach space $X$. For $n=1,2,3, \ldots$, the Minkowski functional \|\|$_{n}$ of the set $U_{n}=2^{n} W+2^{-n} B_{X}$ is a norm equivalent to \|\| . Define, for $x \in X, \quad\|i \mid\|=\left(\sum_{n=1}^{\infty}\|x\|_{n}^{2}\right)^{\frac{1}{2}}$; let $Z=\{x \in X:\|x\|<\infty\}$ and let $j$ denote the identity embedding of $Z$ into $X$; then $(Z,\|\| \|)$ is a Banach space and $j$ is continuous [1, lemma 1 , (ii)].

Theorem. Let $X, Y$ be Banach spaces and let $T \in \mathrm{L}(X, Y)$. Then the following properties are equivalent:

(i) $T \in \operatorname{Ro}(X, Y)$

(ii) $T \in \mathrm{Op}\left(N \ell_{1}\right)(X, Y)$.

Proof: (i) $\Longrightarrow$ (ii). Suppose that $T \in \operatorname{Ro}(X, Y)$. With reference to [1], put $W=T\left(B_{X}\right), \quad M=\{y \in Y:|\|y \mid\|<\infty\}$ and let $j$ denote the identity embedding of $M$ into $Y$. Let $\left(y_{n}\right)$ be a sequence of elements of $W$, then $\left(y_{n}\right)$ has a subsequence $\left(y_{n_{j}}\right)$ such that $\left(J_{Y} y_{n_{j}}\right)$ is $\left(Y^{\prime \prime}, Y^{\prime}\right)$-convergent in $Y^{\prime \prime}$. Hence $J_{Y} W$ is $\sigma\left(Y^{\prime \prime}, Y^{\prime}\right)$-sequentially compact in $Y^{\prime \prime}$ and so by virtue of $[1$, lemma 1 , (xii)], $J_{M} B_{M}$ is $\sigma\left(M^{\prime \prime}, M^{\prime}\right)$-sequentially compact in $M^{\prime \prime}$. This implies that $M \in N \ell_{1}$.

The operators $j^{-1} T: X \rightarrow M$ and $j: M \longrightarrow Y$ provide the required factorization.

(ii) $\Longrightarrow$ (i). It is trivial.

Remark 1. In a 1980 paper [4], S. Heinrich showed that if $T \in \operatorname{Ro}(X, Y)$ then $T \in \mathrm{Op}\left(N \ell_{1}\right)(X, Y)$ proving that the operator ideal of all Rosenthal operators is injective surjective and satisfy the $\Sigma_{p}$-condition for $1<p<\infty$. That is, for arbitrary Banach spaces $X_{n}, Y_{n}, n \in N$, the followings holds:

If $T \in \mathrm{T}\left(\left(\Sigma X_{n}\right)_{p},\left(\Sigma Y_{n}\right)_{p}\right)$, and $Q_{n} T P_{m} \in \operatorname{Ro}\left(X_{m}, Y_{n}\right), n, m \in N$ then $T \in$ $\operatorname{Ro}\left(\left(\Sigma X_{n}\right),\left(\Sigma Y_{n}\right)\right)$ where $P_{m}$ and $Q_{n}$ denote the projections of $\left(\Sigma X_{n}\right)_{p},\left(\Sigma Y_{n}\right)_{p}$ onto the coordinates $X_{m}$ and $Y_{n}$, respectively.

Remark 2. Other characterizations of the Rosenthal operators were obtained by A. Fakhoury [3].

\section{References}

1. W.J. DaVIS, T. FIgIEL, W.B. JOHNSON AND A. PELCZYNSKY, Factoring weakly compact operators, J. Funct. Anal. 17 (1974), 311-327.

2. L.E. DOR, On sequences spanning a complex $\ell_{1}$ - space, Proc. Amer. Math. Soc. 47 (1975), 515-516.

3. H. FAKHouRY, Sur les espaces de Banach ne contenant pas $\ell_{I}(N)$, Math. Scand. 41 (1977), 277-289.

4. S. HEINRICH, Closed operator ideals and Interpolation, J. Functional Analysis 35 (1980), 397-411. 
5. H.HOWARD, A generalization of reflexive Banach spaces and weakly compact operators, Com. Math. Univ. Carolina 13 (1972), 673-684.

6. A. PIETSCH, Operator Ideals, North-Holland (1980).

7. O.J. REINOV, Certain classes of continuous linear operators, Math. Notes 23, no. 2 (1978), 154-159.

8. H. P. ROSENTHAL, A characterization of Banach spaces containing $\ell^{1}$, Proc. Nat. Acad. Sci. USA 71 (1974), 2411-2413.

\author{
Departamento Teoria de Funciones \\ Facuitad de Ciencias \\ Universidad de Santander \\ Santander, SPAIN.
}

Rebut el 18 de Maig de 1987 